\title{
Application and Interpretation of High-resolution Manometry for Pharyngeal Dysphagia
}

\author{
Ju Seok Ryu, ${ }^{1 *}$ Donghwi Park ${ }^{1}$ and Jin Young Kang ${ }^{2}$ \\ ${ }^{1}$ Department of Rehabilitation Medicine, Seoul National University Bundang Hospital, Seoul National University College of Medicine, Seongnam, \\ Gyeonggi-do, Korea; and 'Department of Rehabilitation Medicine, St. Paul's Hospital, College of Medicine, The Catholic University, Seoul, \\ Korea
}

The pharyngeal phase of swallowing is a complex event consisted with subsequent muscular contractions and pressure generation to move a bolus from the mouth to the esophagus. Recently, high-resolution impedance manometry (HRIM) was developed and used for the evaluation of pharyngeal dysphagia. Although HRIM provides precise pharyngeal pressure information, it has yet to be used as part of routine clinical practice for the assessment of dysphagia. The main reasons are thought to be that the test method and result interpretation are not easily applicable and standardized. The anatomical landmarks for HRIM parameters are velopharynx, tongue base, epiglottis, low pharynx, and upper esophageal sphincter. With HRIM, the pressure and timing data could be obtained at a precise anatomical structure. In the present review, we will review how to apply HRIM for the evaluation of pharyngeal dysphagia, including the interpretation of its parameters.

(J Neurogastroenterol Motil 2015;21:283-287)

\section{Key Words}

Deglutition disorders; Diagnosis; Manometry; Pharynx

\section{Introduction}

Dysphagia means difficulty in swallowing, which may be followed by aspiration and inadequate nutrition. Dysphagia results from diverse etiologies, and its likelihood of occurrence increases with age. Pharyngeal swallow is a complex event that requires subsequent muscular contractions and pressure generation to move a bolus from the mouth to the esophagus. ${ }^{1}$

Until now, a videofluoroscopic swallow study (VFSS), an $\mathrm{X}$-ray-based analysis of swallowing, has been a gold standard in analyzing the swallowing function in patients with dysphagia. However, VFSS could only evaluate the movement of anatomic structures and bolus, and could not evaluate the generated phar-

Received: January 14, 2015 Revised: February 16, 2015 Accepted: February 18, 2015

(c) This is an Open Access article distributed under the terms of the Creative Commons Attribution Non-Commercial License (http://creativecommons. org/licenses/by-nc/3.0) which permits unrestricted non-commercial use, distribution, and reproduction in any medium, provided the original work is properly cited.

${ }^{*}$ Correspondence: Ju Seok Ryu, MD, PhD

Department of Rehabilitation Medicine, Seoul National University Bundang Hospital, Seoul National University College of Medicine, 82 Gumi-ro 173 Beon-gil, Bundang-gu, Seongnam, Gyeonggi-do 463-707, Korea

Tel: +82-31-787-7739, Fax: +82-31-787-4051, E-mail: jseok337@snu.ac.kr

Financial support: This research was supported by Basic Science Research Program through the National Research Foundation of Korea (NRF) funded by the Ministry of Science, ICT, and Future Planning (NRF-2013R1A1A1004622).

Conflicts of interest: None.

Author contributions: Ju Seok Ryu prepared manuscript and set up high-resolution manometry; Donghwi Park and Jin Young Kang assisted data collection and analysis.

ORCID: Ju Seok Ryu, http://orcid.org/0000-0003-3299-3038. 
yngeal pressure. There exists a correlation between the abnormalities in generating adequate pharyngeal pressure and developing pharyngeal dysphagia; therefore new methods to evaluate and analyze the pharyngeal pressure events are required to reveal the underlying pathophysiology of dysphagia.

As conventional manometry uses hydrostatic pressure, only the limited sensors and positions (supine) were allowed; therefore, its application to pharyngeal dysphagia was nearly impossible. Recently, high-resolution impedance manometry (HRIM) was developed and used to evaluate pharyngeal dysphagia. HRIM has pressure sensors at 1-2 cm intervals and uses a solid catheter, which enables the evaluation at the sitting position. It also has improved sensitivity, reliability, and accuracy. ${ }^{2}$ Although HRIM provides precise pharyngeal pressure information, it has yet to be applied to routine clinical practice as the assessment method for dysphagia. The main reason may be that the test method and result interpretation are not easily applicable and standardized. In the present review, we will discuss the methods to use HRIM, including the interpretation of its parameters, to assist in the evaluation of pharyngeal dysphagia.

A

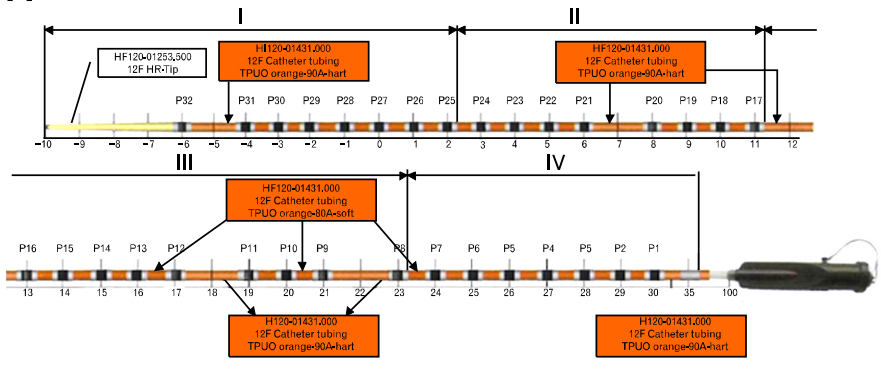

C

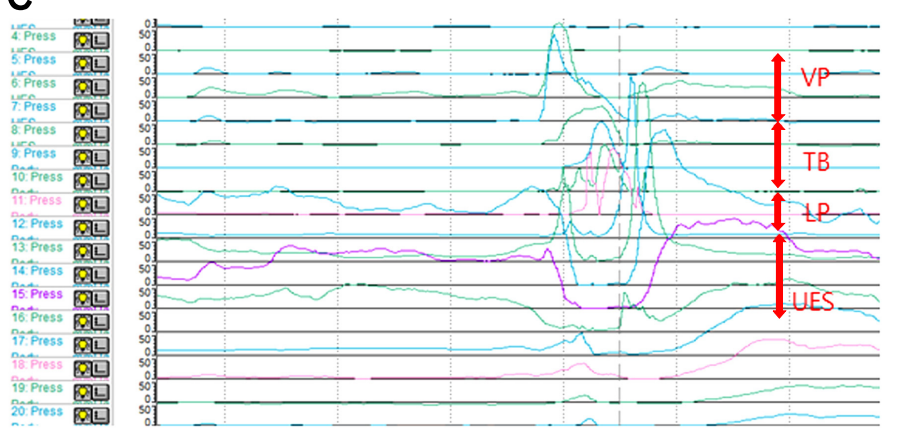

\section{Test Equipment}

A solid HRIM that can measure the rapidly changing pressures along the entire length of the pharynx is used for all data collection (INSIGHT HRIM; Sandhill Scientific Inc, Highlands Ranch, CO, USA). ${ }^{3}$ The manometric catheter uses 32 circumferential pressure sensors that are spaced $1-2 \mathrm{~cm}$ apart. The diagram of the manometric catheter is represented in Figure 1A. In most areas of the manometric catheter, the intervals of the sensors are $1 \mathrm{~cm}$ apart, while $2 \mathrm{~cm}$ apart only in 5 areas; therefore, the capable length of measure is $36 \mathrm{~cm}$ (Fig. 1A). The system is calibrated to record the pressures between 0 and $100 \mathrm{mmHg}$ in accordance to the manufacturer's specifications.

\section{Test Procedure}

The examination is performed at a neutral head and sitting position. Participants are instructed not to eat for 4 hours and not drink liquids for 2 hours prior to testing in order to avoid any potential confounding effects of satiety. ${ }^{4} \mathrm{~A} 10 \%$ lidocaine spray is
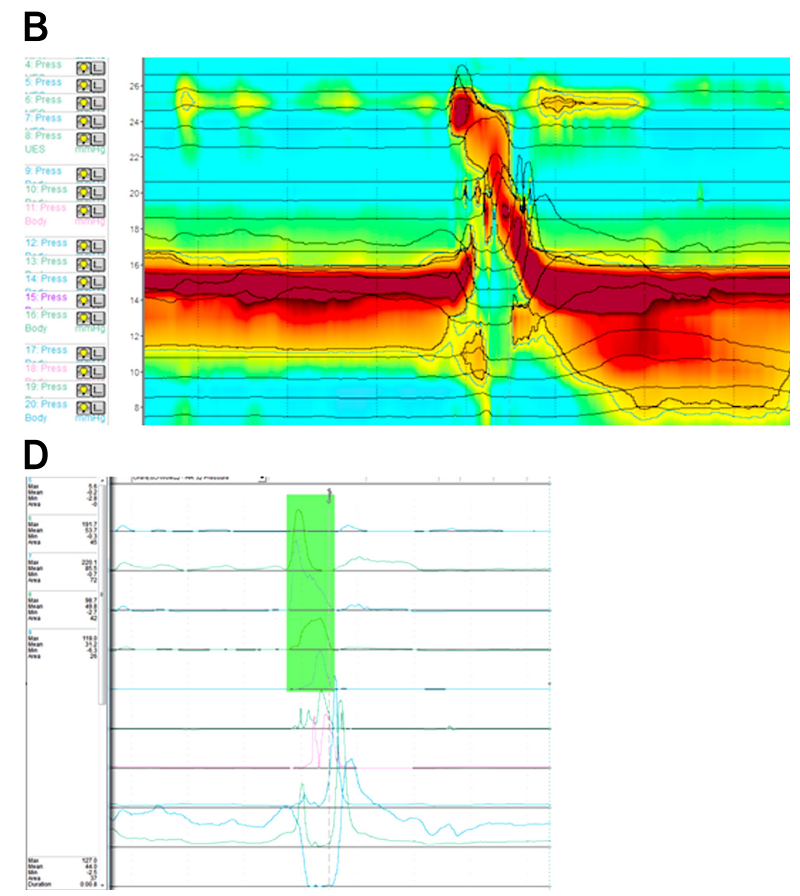

Figure 1. (A) A diagram of the manometric catheter. This catheter uses 32 circumferential pressure sensors spaced $1-2 \mathrm{~cm}$ apart. In most areas of the manometric catheter, the intervals of sensors are $1 \mathrm{~cm}$ apart, while $2 \mathrm{~cm}$ apart in 5 areas. After we disable the unrelated channels and (B) close "ClouseVIEW," (C) "Waveform" is shown. The red arrows show the velopharynx, tongue base, low pharynx, and upper esophageal sphincter. (D) We measure the maximal, mean, minimal pressure, and area (integral) in the selected area. "ClouseVIEW" and "Waveform" are display modes of BioVIEW ANALYSIS software (Version 5.6.3.0). 
applied through the nasal passage. Manometric catheter is lubricated with a $2 \%$ lidocaine jelly to ease the passage of the catheter through the pharynx. Once the catheter is positioned within the pharynx, participants rest for 5-10 minutes for adaptation prior to swallowing. The areas of interest are from the velopharynx to the upper esophageal sphincter (UES). Therefore, manometric catheters should be pulled back $10 \mathrm{~cm}$ from HRIM standard protocol to encompass the area of interest (HRIM-modified protocol; Fig. $1 \mathrm{~B}$ and $1 \mathrm{C}){ }^{5}$ Usually the area of interest is within 10 $\mathrm{cm}$.

Then the participants swallow $5 \mathrm{~mL}$ of saline or water 2 times. As the volume is shown to influence the parameters of HRIM, it should be controlled throughout the examination of HRIM. Moreover, repetitive swallowing with different texture is not required, as texture is not shown to affect the results. ${ }^{6,7}$ In our opinion, $5 \mathrm{~mL}$ thin liquid (water or normal saline) is sufficient.

\section{Analysis and Interpretations}

Data are extracted using BioVIEW ANALYSIS software (Sandhill Scientific, Version 5.6.3.0). As the HRIM measures the changes of pressures at specific anatomic structures, we need to define these structures for higher reliability. The anatomical landmarks for HRIM parameters are velopharynx (VP), tongue base (TB), epiglottis, low pharynx (LP), and UES (Fig. 1C). ${ }^{2,4}$ The maximal and minimal pressures, the area integral to pressure peak, and the timing intervals between the variables are measured (Fig. 1D).

\section{Velopharynx and Tongue Base}

VP is defined anteriorly by the soft palate and posteriorly by the posterior pharyngeal wall. TB is defined as a portion posterior and superior to the tongue. VP and TB pressure peaks are easily detected in Figure $1 \mathrm{~B}$ and $1 \mathrm{C}$.

Anatomically, TB is located slightly higher than the epiglottis. We can observe a prominent high peak with short duration, which is summating with the TB peak pressure in Figure $2 \mathrm{~A}[1]$. As the epiglottis tilts, it strikes the manometric catheter (Fig. 2B and 2C) and a peak with high amplitude and short duration is created (Fig. 2A [1]). This peak changes the maximal peak pressure, but a minimal degree of the area. Therefore, the higher pressure peak of TB should be measured at other channels that are nearby. We recommend selecting a TB channel that shows the highest amplitude and area between VP and epiglottis. This channel is usually located 1 channel higher than the peak of

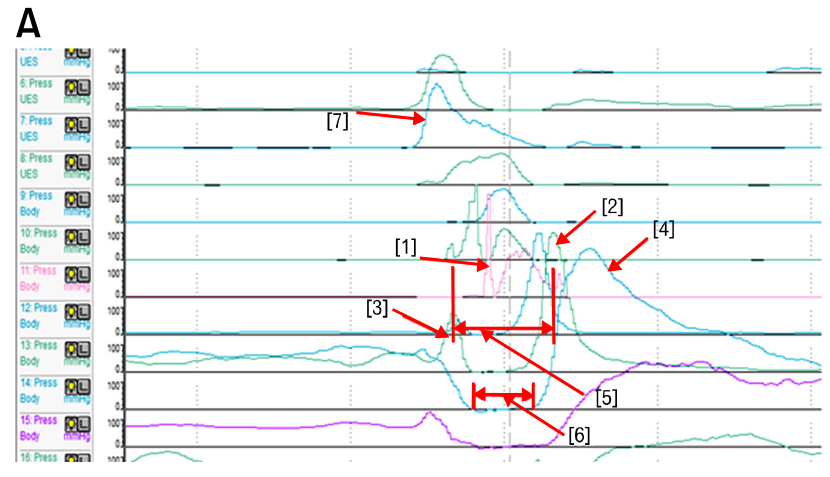

B

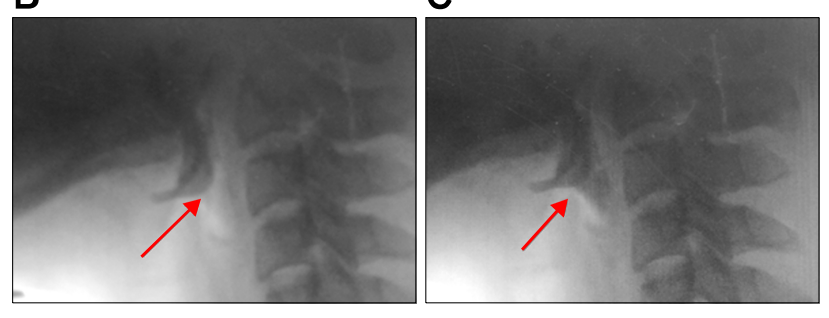

Figure 2. (A) Shows the individual peaks in the areas of interest. Each peak shows [1] tilting of epiglottis, [2] low pharyngeal peak, [3] UES peak, [4] pre-upper esophageal sphincter (UES) peak, [5] UES activity time and [6] Nadir UES duration. The [7] peak shows the simultaneous contraction of the velopharynx and tongue base. (B) Shows the contact of bolus with epiglottis. (C) Shows the tilting of epiglottis. At this time, the tilted epiglottis strikes the manometric catheter, resulting in peak [1].

the epiglottis.

As the intervals of the channels are 1 to $2 \mathrm{~cm}$, these epiglottis peaks are sometimes not visualized. In these cases, we can adjust the manometric catheter slightly up and down to visualize the epiglottis peak or select the TB channel that show the highest peak between VP and LP without considering the epiglottis peak. The epiglottis peak can be ignored according to the purpose of the evaluation.

\section{Pharyngeal Constrictor and Upper Esophageal Sphincter}

We can observe the narrow peristaltic waves and the last broad peak in Figure 2A [2] and 2A [4]. Anatomically, pharyngeal constrictors are comprised of the superior, middle, and inferior pharyngeal constrictors. In addition, the upper esophageal sphincters are composed of the inferior pharyngeal constrictor muscle and cricopharyngeus muscle. ${ }^{8}$ The pharyngeal constrictor muscle consists mainly of the faster twitch type II fiber, and the cricopharyngeus muscle is primarily composed of the highly oxi- 
dative, slow twitch type I fiber. Therefore, the narrow peaks represent the pharyngeal constrictor muscles, and the broad last peak represents the cricopharyngeus muscle. ${ }^{9,10}$ For a comparison between these peaks, we defined one channel that show the highest peaks among the shallow peaks as the "low pharyngeal peak" (LP peak, Fig. 2A [2]), while we defined the broad peak as the "UES peak" (Fig. 2A [4]).

In summary, the peaks of the pharyngeal constrictors are measured between TB and UES. LP peak represents the maximal pressure of the pharyngeal constrictors. Usually, the lowest wave that represents the inferior pharyngeal constrictor muscle is the highest wave. At the region of UES, we can also measure the pre-UES maximal pressure (Fig. 2A [3]), and minimal UES pressure.

\section{The Time Intervals}

The rise time and duration of the $\mathrm{VP}$ and $\mathrm{TB}$ regions can be measured. We can also measure the interval between the pre-UES peak and post-UES peak (UES activity time; Fig. 2A [5]) and the duration between the lowest areas (Nadir UES duration; Fig. 2A [6]).,

We can also measure the time intervals between $\mathrm{VP}$ onset and epiglottis peak, VP onset and TB onset, VP peak and epiglottis peak, VP peak and TB peak, VP onset and Pre-UES peak, and VP onset and Post-UES peak. Using the results obtained, we can calculate the rate of pressure generated in VP and TB (maximal peak amplitude/rise time) and the pressure wave velocity (the distance from the VP pressure peak to the LP peak/the time lapse between these 2 points). ${ }^{1}$

As we mentioned previously, some individuals do not show an epiglottis peak. Therefore, this parameter cannot be applied to all patients; if it is necessary to evaluate this parameter, we should adjust the manometric catheter slightly up and down to visualize this peak.

\section{Limitations of High-resolution Manometry}

Although close intervals between the sensors are an advantage of HRIM compared to the conventional manometry, it can also be a limitation. As the pharyngeal structures are closely located, 1 or $2 \mathrm{~cm}$ of intervals prevent detailed examination, especially the epiglottis and the junction between the inferior pharyngeal constrictor muscle and cricopharyngeus muscles. In addition, the channel can detect a contraction of adjacent anatomical structure in both sides. The wave in Figure 2A [7] shows a contraction of $\mathrm{VP}$ and TB simultaneously. Although the maximal amplitude represents that of $\mathrm{VP}$, the area is difficult to measure due to the summation of VP and TB. Therefore, agreements between the raters and proper channel selections are required. If new equipment with a continuous sensor is developed, more accurate analysis may be possible.

The second limitation is that it cannot be analyzed by an automatic analysis program. Previously, an automated analysis of the pharyngeal pressure with HRIM was attempted. ${ }^{2}$ However, an automatic analysis is not easily applicable due to the summation of adjacent anatomical structures. Therefore, a manual and a time consuming analysis is inevitable. However, it is worth noting that experienced examiners can analyze within 5 minutes per patient.

The third limitation is the position of the HRIM catheter. As pharyngeal structures are not round, the manometric catheter could be located on the lateral side (especially in swallowing maneuvers such as head rotation or tilting). In these cases, the HRIM catheter could not reflect the real pressure, especially the rotated or tilted position. ${ }^{11}$

The fourth limitation is that HRIM could not represent the presence of an aspiration. Hence, VFSS may not be replaceable by HRIM, but rather be assisted by HRIM. Previous research found swallows in patients with suspected aspiration have lower peak pressure, higher pressure at nadir impedance, longer flow interval and shorter time from nadir impedance to peak pressure than asymptomatic controls. ${ }^{12}$ But further study is required to be used for clinical practice.

The fifth limitation is that HRM could not represent the degree of pharyngeal residue in patients with oropharyngeal dysphagia. Omari et al. reported that combined manometry and multichannel intraluminal impedance facilitated prediction of pharyngeal residue after swallowing ${ }^{13}$ and other studies reported that a simple analysis of the impedance contour pattern (linear vs. stasis) could reveal pharyngeal residue. ${ }^{5,14}$ As these analyses are qualitative rather than quantative, follow up studies are required for clinical application.

\section{Conclusions}

HRIM can provide precise pressure events and timing data for pharyngeal swallowing. With the development of HRIM parameters for pharyngeal dysphagia, its interpretation and applicability can be improved. As HRIM could provide quantitative 
analysis of the pressure events of pharyngeal structures, this equipment may be helpful for the diagnosis and monitoring of pharyngeal dysphagia and provide comprehensive understanding of the swallowing process.

\section{References}

1. Mielens JD, Hoffman MR, Ciucci MR, McCulloch TM, Jiang JJ. Application of classification models to pharyngeal high-resolution manometry. J Speech Lang Hear Res 2012;5 5:892-902.

2. Mielens JD, Hoffman MR, Ciucci MR, Jiang JJ, McCulloch TM. Automated analysis of pharyngeal pressure data obtained with high-resolution manometry. Dysphagia 2011;26:3-12.

3. Fox MR, Bredenoord AJ. Oesophageal high-resolution manometry: moving from research into clinical practice. Gut 2008;57:405-423.

4. McCulloch TM, Hoffman MR, Ciucci MR. High-resolution manometry of pharyngeal swallow pressure events associated with head turn and chin tuck. Ann Otol Rhinol Laryngol 2010;119:369-376.

5. Lee TH, Lee JS, Park JW, et al. High-resolution impedance manometry facilitates assessment of pharyngeal residue and oropharyngeal dysphagic mechanisms. Dis Esophagus 2014;27:220-229.

6. Hoffman MR, Ciucci MR, Mielens JD, Jiang JJ, McCulloch TM. Pharyngeal swallow adaptations to bolus volume measured with high-resolution manometry. Laryngoscope 2010;120:2367-2373.

7. Lee TH, Ryu JS. High resolution manometry of pharyngeal swallow pressure events associated with food viscosity. ACRM (American congress of rehabilitation medicine) 91th Annual Conference 2014, October, Toronto, ON, Canada.

8. Singh S, Hamdy S. The upper oesophageal sphincter. Neurogastroenterol Motil 2005;17(suppl 1):3-12.

9. Bonington A, Mahon M, Whitmore I. A histological and histochemical study of the cricopharyngeus muscle in man. J Anat 1988; 56:27-37.

10. Mu L, Sanders I. Neuromuscular compartments and fiber-type regionalization in the human inferior pharyngeal constrictor muscle. Anat Rec 2001;264:367-377.

11. Takasaki K, Umeki H, Kumagami H, Takahashi H. Influence of head rotation on upper esophageal sphincter pressure evaluated by high-resolution manometry system. Otolaryngol Head Neck Surg 2010;142:214-217.

12. Omari TI, Dejaeger E, van Beckevoort D, et al. A method to objectively assess swallow function in adults with suspected aspiration. Gastroenterology 2011;140:1454-1463.

13. Omari TI, Dejaeger E, Van Beckevoort D, et al. A novel method for the nonradiological assessment of ineffective swallowing. Am J Gastroenterol 2011;106:1796-1802.

14. Lee TH, Lee JS, Hong SJ, et al. Impedance analysis using high-resolution impedance manometry facilitates assessment of pharyngeal residue in patients with oropharyngeal dysphagia. J Neurogastroenterol Motil 2014;20:362-370. 